University of Texas at El Paso

ScholarWorks@UTEP

4-2008

\title{
Fast Algorithms for Uncertainty Propagation, and Their Applications to Structural Integrity
}

Andrzej Pownuk

The University of Texas at El Paso, ampownuk@utep.edu

Jakub Cerveny

Jerald Brady

The University of Texas at El Paso, jjbrady@utep.edu

Follow this and additional works at: https://scholarworks.utep.edu/cs_techrep

Part of the Computer Engineering Commons

Comments:

Technical Report: UTEP-CS-08-23

Published in Proceedings of the 27th International Conference of the North American Fuzzy

Information Processing Society NAFIPS'2008, New York, New York, May 19-22, 2008.

\section{Recommended Citation}

Pownuk, Andrzej; Cerveny, Jakub; and Brady, Jerald, "Fast Algorithms for Uncertainty Propagation, and Their Applications to Structural Integrity" (2008). Departmental Technical Reports (CS). 90.

https://scholarworks.utep.edu/cs_techrep/90

This Article is brought to you for free and open access by the Computer Science at ScholarWorks@UTEP. It has been accepted for inclusion in Departmental Technical Reports (CS) by an authorized administrator of ScholarWorks@UTEP. For more information, please contact Iweber@utep.edu. 


\title{
Fast Algorithms for Uncertainty Propagation, and Their Applications to Structural Integrity
}

\author{
Andrzej Pownuk, Jakub Cerveny, and Jerald J. Brady \\ University of Texas at El Paso \\ E1 Paso, TX 79968 \\ Contact email ampownuk@utep.edu
}

\begin{abstract}
In many practical situations, we need to know how uncertainty propagates through data processing algorithms, i.e., how the uncertainty in the inputs affects the results of data processing. This problem is important for all types of uncertainty: probabilistic, interval, and fuzzy. From the computational viewpoint, however, this problem is much more complex for interval and fuzzy uncertainty. Therefore, for these types of uncertainty, it is desirable to design faster algorithms.

In this paper, we describe faster algorithms for two practically important situations:

- linearization situations, when the approximation errors are small and therefore, the data processing algorithms can be replaced by a linear function, and

- monotonic situations, when the dependence of the result $y$ of data processing on each of the inputs $x_{1}, \ldots, x_{n}$ is either monotonically increasing or monotonically decreasing.
\end{abstract}

\section{Practical NeEd FOR UnCERTAinty Propagation}

In many practical situations, we are interested in the value of a quantity $y$ which is difficult or even impossible to measure directly. To estimate this difficult-to-measure quantity $y$, we measure or estimate related easier-to-measure quantities $x_{1}, \ldots, x_{n}$ which are related to the desired quantity $y$ by a known relation $y=f\left(x_{1}, \ldots, x_{n}\right)$. Then, we apply the relation $f$ to the estimates $\widetilde{x}_{1}, \ldots, \widetilde{x}_{n}$ for $x_{i}$ and produce an estimate $\widetilde{y}=f\left(\widetilde{x}_{1}, \ldots, \widetilde{x}_{n}\right)$ for the desired quantity $y$.

In the simplest cases, the relation $f\left(x_{1}, \ldots, x_{n}\right)$ may be an explicit expression: e.g., if we know the current $x_{1}$ and the resistance $x_{2}$, then we can measure the voltage $y$ by using Ohm's law $y=x_{1} \cdot x_{2}$. In many practical situations, the relation between $x_{i}$ and $y$ is much more complicated: the corresponding algorithm $f\left(x_{1}, \ldots, x_{n}\right)$ is not an explicit expression, but a complex algorithm for solving an appropriate non-linear equation (or system of equations).

Estimates are never absolutely accurate:

- measurements are never absolutely precise, and

- expert estimates can only provide approximate values of the directly measured quantities $x_{1}, \ldots, x_{n}$.

In both cases, the resulting estimates $\widetilde{x}_{i}$ are, in general, different from the actual (unknown) values $x_{i}$. Due to these estimation errors $\Delta x_{i} \stackrel{\text { def }}{=} \widetilde{x}_{i}-x_{i}$, even if the relation $f\left(x_{1}, \ldots, x_{n}\right)$ is exact, the estimate $\widetilde{y}=f\left(\widetilde{x}_{1}, \ldots, \widetilde{x}_{n}\right)$ is different from the actual value $y=f\left(x_{1}, \ldots, x_{n}\right): \Delta y \stackrel{\text { def }}{=} \widetilde{y}-y \neq 0$.

(In many situations, when the relation $f\left(x_{1}, \ldots, x_{n}\right)$ is only known approximately, there is an additional source of the approximation error in $y$ caused by the uncertainty in knowing this relation.)

It is therefore desirable to find out how the uncertainty $\Delta x_{i}$ in estimating $x_{i}$ affects the uncertainty $\Delta y$ in the desired quantity, i.e., how the uncertainties $\Delta x_{i}$ propagate via the algorithm $f\left(x_{1}, \ldots, x_{n}\right)$.

\section{Propagation of PRobabilistic UnCERTAinty}

Often, we know the probabilities of different values of $\Delta x_{i}$. For example, in many cases, we know that the approximation errors $\Delta x_{i}$ are independent normally distributed with zero mean and known standard deviations $\sigma_{i}$; see, e.g., [14].

In this case, we can use known statistical techniques to estimate the resulting uncertainty $\Delta y$ in $y$. For example, since we know the probability distributions, we can simulate them in the computer, i.e., use the Monte-Carlo simulation techniques to get a sample population $\Delta y^{(1)}, \ldots, \Delta y^{(N)}$ of the corresponding errors $\Delta y$. Based on this sample, we can then estimate the desired statistical characteristics of the desired approximation error $\Delta y$.

\section{PROPAGATION OF INTERVAL UNCERTAINTY}

In many other practical situations, we do not know these probabilities, we only know the upper bounds $\Delta_{i}$ on the (absolute values of) the corresponding measurement errors $\Delta x_{i}:\left|\Delta x_{i}\right| \leq \Delta$.

In this case, based on the known approximation $\widetilde{x}_{i}$, we can conclude that the actual (unknown) value of $i$-th auxiliary quantity $x_{i}$ can take any value from the interval

$$
\mathbf{x}_{i}=\left[\widetilde{x}_{i}-\Delta_{i}, \widetilde{x}_{i}+\Delta_{i}\right]
$$

To find the resulting uncertainty in $y$, we must therefore find the range $\mathbf{y}=[\underline{y}, \bar{y}]$ of possible values of $y$ when $x_{i} \in \mathbf{x}_{i}$ :

$$
\begin{gathered}
\mathbf{y}=f\left(\mathbf{x}_{1}, \ldots, \mathbf{x}_{n}\right) \stackrel{\text { def }}{=} \\
\left\{f\left(x_{1}, \ldots, x_{n}\right) \mid x_{1} \in \mathbf{x}_{1}, \ldots, x_{n} \in \mathbf{x}_{n}\right\} .
\end{gathered}
$$

Computations of this range under interval uncertainty is called interval computations; see, e.g., [3], [4].

The corresponding computational problems are, in general, NP-hard [8]. Crudely speaking, this means that, in general, such problems require a large amount of computation time and that therefore faster methods are needed. 


\section{PRopagation OF FUZZY UNCERTAINTY}

In many practical situations, the estimates $\widetilde{x}_{i}$ come from experts. Experts often describe the inaccuracy of their estimates in terms of imprecise words from natural language, such as "approximately 0.1 ", etc. A natural way to formalize such words is to use special techniques developed for formalizing this type of estimates - specifically, the technique of fuzzy logic; see, e.g., [5], [11].

In this technique, for each possible value of $x_{i} \in \mathbf{x}_{i}$, we describe the degree $\mu_{i}\left(x_{i}\right)$ to which this value is possible. For each degree of certainty $\alpha$, we can determine the set of values of $x_{i}$ that are possible with at least this degree of certainty the $\alpha$-cut $\mathbf{x}_{i}(\alpha)=\{x \mid \mu(x) \geq \alpha\}$ of the original fuzzy set. Vice versa, if we know $\alpha$-cuts for every $\alpha$, then, for each object $x$, we can determine the degree of possibility that $x$ belongs to the original fuzzy set [2], [5], [9], [10], [11]. A fuzzy set can be thus viewed as a nested family of its (interval) $\alpha$-cuts.

We already know how to propagate interval uncertainty. Thus, to propagate this fuzzy uncertainty, we can therefore consider, for each $\alpha$, the fuzzy set $y$ with the $\alpha$-cuts

$$
\mathbf{y}(\alpha)=f\left(\mathbf{x}_{1}(\alpha), \ldots, \mathbf{x}_{1}(\alpha)\right) ;
$$

see, e.g., [2], [5], [9], [10], [11]. So, from the computational viewpoint, the problem of propagating fuzzy uncertainty can be reduced to several interval propagation problems.

\section{NEED FOR FASTER ALGORITHMS FOR UNCERTAINTY PROPAGATION}

Summarizing the above analysis, we can conclude that in principle, we need to consider three possible types of uncertainty propagation: situations when we propagate probabilistic, interval, and fuzzy uncertainty.

For probabilistic uncertainty, there exist reasonable efficient uncertainty propagation algorithms such as Monte-Carlo simulations. In contrast, the problems of propagating interval and fuzzy uncertainty are, in general, computationally difficult. It is therefore desirable to design faster algorithms for propagating interval and fuzzy uncertainty.

The computational problem of propagating fuzzy uncertainty can be naturally reduced to the problem of propagating interval uncertainty. Because of this reduction, in the following text, we will mainly concentrate on faster algorithms for propagating interval uncertainty. Applications of the above algorithms to different structural integrity problems are described in [12], [13].

\section{TWO SITUATIONS}

In this paper, we describe faster algorithms for two practically important situations:

- linearization situations, when the approximation errors are small and therefore, the data processing algorithms can be replaced by a linear function, and

- monotonic situations, when the dependence of the result $y$ of data processing on each of the inputs $x_{1}, \ldots, x_{n}$ is either monotonically increasing or monotonically decreasing.

\section{LINEARIZATION SITUATIONS: DESCRIPTION}

Due to the approximation errors $\Delta x_{i}=\widetilde{x}_{i}-x_{i}$, the unknown (actual) values $x_{i}=\widetilde{x}_{i}-\Delta x_{i}$ of the input quantities $x_{i}$ are, in general, different from the approximate estimates $\widetilde{x}_{i}$. In many practical situations, the approximation errors $\Delta x_{i}$ are small - e.g., when the approximations are obtained by reasonably accurate measurements. In such situations, we can ignore terms which are quadratic (and of higher order) in $\Delta x_{i}$.

\section{LINEARIZATION SITUATIONS: ANALYSIS}

In the above situations, we can expand the expression for

$$
\begin{gathered}
\Delta y=\widetilde{y}-y=f\left(\widetilde{x}_{1}, \ldots, \widetilde{x}_{n}\right)-f\left(x_{1}, \ldots, x_{n}\right)= \\
f\left(\widetilde{x}_{1}, \ldots, \widetilde{x}_{n}\right)-f\left(\widetilde{x}_{1}-\Delta x_{1}, \ldots, \widetilde{x}_{n}-\Delta x_{n}\right)
\end{gathered}
$$

in Taylor series in $\Delta x_{i}$ and keep only the linear terms in this expansion. In this case, we get

$$
\Delta y=c_{1} \cdot \Delta x_{1}+\ldots+c_{n} \cdot \Delta x_{n},
$$

where we denoted

$$
c_{i} \stackrel{\text { def }}{=} \frac{\partial f}{\partial x_{i}}\left(\widetilde{x}_{1}, \ldots, \widetilde{x}_{n}\right) .
$$

For a linear function, the largest possible value of $\Delta y$ is obtained when each of the variables $\Delta x_{i} \in\left[-\Delta_{i}, \Delta_{i}\right]$ attains:

- either its largest value $\Delta_{i}$ (when $\left.c_{i} \geq 0\right)$

- or its smallest value $-\Delta_{i}$ (when $\left.c_{i}<0\right)$.

In both cases, the largest possible value of the corresponding term in $\Delta y$ is equal to $\left|c_{i}\right| \cdot \Delta_{i}$. Thus, the largest possible value of $\Delta y$ is equal to

$$
\Delta=\left|c_{1}\right| \cdot \Delta_{1}+\ldots+\left|c_{n}\right| \cdot \Delta_{n} .
$$

Similarly, the smallest possible value of $\Delta y$ is obtained when each of the variables $\Delta x_{i} \in\left[-\Delta_{i}, \Delta_{i}\right]$ attains

- either its smallest value $-\Delta_{i}$ (when $\left.c_{i} \geq 0\right)$

- or its largest value $\Delta_{i}$ (when $c_{i}<0$ ).

In both cases, the smallest possible value of the corresponding term in $\Delta y$ is equal to $-\left|c_{i}\right| \cdot \Delta_{i}$. Thus, the smallest possible value of $\Delta y$ is equal to

$$
-\Delta=-\left|c_{1}\right| \cdot \Delta_{1}-\ldots-\left|c_{n}\right| \cdot \Delta_{n} .
$$

Can can we transform these natural formulas into an algorithm? Due to the linearization assumption, we can estimate each partial derivative $c_{i}$ as

$$
c_{i} \approx \frac{f\left(\widetilde{x}_{1}, \ldots, \widetilde{x}_{i-1}, \widetilde{x}_{i}+h_{i}, \widetilde{x}_{i+1}, \ldots, \widetilde{x}_{n}\right)-\widetilde{y}}{h_{i}}
$$

for some small values $h_{i}$. So, we arrive at the following algorithm. 


\section{LINEARIZATION SITUATIONS: ALGORITHM}

To compute the range $\mathbf{y}$ of $y$, we do the following.

- First, we apply the algorithm $f$ to the original estimates $\widetilde{x}_{1}, \ldots, \widetilde{x}_{n}$, resulting in the value $\widetilde{y}=f\left(\widetilde{x}_{1}, \ldots, \widetilde{x}_{n}\right)$.

- Second, for all $i$ from 1 to $n$, we compute $f\left(\widetilde{x}_{1}, \ldots, \widetilde{x}_{i-1}, \widetilde{x}_{i}+h_{i}, \widetilde{x}_{i+1}, \ldots, \widetilde{x}_{n}\right)$ for some small $h_{i}$ and then compute

$$
c_{i}=\frac{f\left(\widetilde{x}_{1}, \ldots, \widetilde{x}_{i-1}, \widetilde{x}_{i}+h_{i}, \widetilde{x}_{i+1}, \ldots, \widetilde{x}_{n}\right)-\widetilde{y}}{h_{i}} .
$$

- Finally, we compute

$$
\Delta=\left|c_{1}\right| \cdot \Delta_{1}+\ldots+\left|c_{n}\right| \cdot \Delta_{n}
$$

and the desired range $\mathbf{y}=[\widetilde{y}-\Delta, \widetilde{y}+\Delta]$.

\section{LINEARIZATION SITUATIONS: COMPUTATIONAL COMPLEXITY}

The main computation time is spent on calling the timeconsuming algorithm $f$. In the above uncertainty propagation algorithm, after one call to $f$ to compute $\widetilde{y}$, we need $n$ calls to $f$ to compute the corresponding partial derivatives $c_{i}$ and then, we can estimate the desired uncertainty $\Delta$ in $y$ by using the above simple formula.

Overall, we thus need $n+1$ calls to the algorithm $f$.

Comment. For large $n$, we can further reduce the number of calls to $f$ if we use a special technique of Cauchy-based Monte-Carlo simulations, which enables us to use a fixed number of calls to $f(\approx 200)$ for all possible values $n$; see, e.g., [6], [7].

\section{MonOtONIC SITUATION WITH KNOWN DIRECTIONS OF MONOTONICITY: DESCRIPTION}

In the previous case, we considered situations in which we can safely ignore terms which are quadratic and of higher order in $\Delta x_{i}$ and in which, therefore, the actual dependence $y=f\left(x_{1}, \ldots, x_{n}\right)$ can be safely approximated by a linear function $\Delta y=\sum_{i=1}^{n} c_{i} \cdot \Delta x_{i}$.

In many practical situations - especially in situations related to expert (fuzzy) estimates when approximation errors may not be small - we can no longer ignore quadratic and higher order terms in $\Delta x_{i}$, so we must consider approximating functions which are more general than the linear ones.

A natural more general class of functions comes from the fact that linear functions are monotonic in each of their variables $\Delta x_{i}$. This class is general enough, since in the generic case, all partial derivatives $\frac{\partial f}{\partial x_{i}}$ are non-zeros at the point $\widetilde{x} \stackrel{\text { def }}{=}\left(\widetilde{x}_{1}, \ldots, \widetilde{x}_{n}\right)$. Hence, these derivatives are also different from 0 in some vicinity of this point - and thus, in this vicinity, the derivatives retain their signs and thus, the function $f$ is monotonic with respect to each of these variables.

In practice, we are indeed often sure that the dependence is monotonic. For example, the Ohm's law $y=f\left(x_{1}, x_{2}\right)=$ $x_{1} \cdot x_{2}$ is not a linear function of its two variables, but it is monotonic for $x_{1}, x_{2} \geq 0$.

\section{MONOTONIC SITUATION WITH KNOWN DIRECTIONS} OF MONOTONICITY: ANALYSIS

For a monotonic function $f\left(x_{1}, \ldots, x_{n}\right)$, the largest possible value over the intervals $\left[\widetilde{x}_{i}-\Delta_{i}, \widetilde{x}_{i}+\Delta_{i}\right]$ is attained when:

- the variables $x_{i}$ w.r.t. which the function $f$ is increasing attain their largest possible values $\widetilde{x}_{i}+\Delta_{i}$, and

- the variables $x_{i}$ w.r.t. which the function $f$ is decreasing attain their smallest possible values $\widetilde{x}_{i}-\Delta_{i}$.

In other words, if we denote, for each input $x_{i}$, the "monotonicity sign" $\varepsilon_{i}$ as $\varepsilon_{i}=1$ if $f$ is increasing in $x_{i}$ and $\varepsilon_{i}=-1$ if $f$ is decreasing in $x_{i}$, then the largest possible value $\bar{y}$ of $y=f\left(x_{1}, \ldots, x_{n}\right)$ is attained when $x_{i}=\widetilde{x}_{i}+\varepsilon_{i} \cdot \Delta_{i}$ for all $i$, i.e.,

$$
\bar{y}=f\left(\widetilde{x}_{1}+\varepsilon_{1} \cdot \Delta_{1}, \ldots, \widetilde{x}_{n}+\varepsilon_{n} \cdot \Delta_{n}\right) .
$$

Similarly, the smallest possible value $y$ of $y=f\left(x_{1}, \ldots, x_{n}\right)$ is attained when $x_{i}=\widetilde{x}_{i}-\varepsilon_{i} \cdot \Delta_{i}$ for all $i$, i.e.,

$$
\underline{y}=f\left(\widetilde{x}_{1}-\varepsilon_{1} \cdot \Delta_{1}, \ldots, \widetilde{x}_{n}-\varepsilon_{n} \cdot \Delta_{n}\right) .
$$

So, if we know with respect to which variables $x_{i}$ it is increasing and with respect to which variables $x_{j}$ this dependence is decreasing, then we can find the desired range $[y, \bar{y}]$ of possible values of $y$ by using the following algorithm.

\section{MONOTONIC SITUATION WITH KNOWN DIRECTIONS OF MONOTONICITY: ALGORITHM}

- First, we compute $\underline{y}$ as

$$
\underline{y}=f\left(\widetilde{x}_{1}-\varepsilon_{1} \cdot \Delta_{1}, \ldots, \widetilde{x}_{n}-\varepsilon_{n} \cdot \Delta_{n}\right) .
$$

- Then, we compute $\bar{y}$ as

$$
\bar{y}=f\left(\widetilde{x}_{1}+\varepsilon_{1} \cdot \Delta_{1}, \ldots, \widetilde{x}_{n}+\varepsilon_{n} \cdot \Delta_{n}\right) .
$$

The resulting range of possible values of $y$ is $[y, \bar{y}]$.

XIV. MONOTONIC SITUATION WITH KNOWN DIRECTIONS OF MONOTONICITY: COMPUTATIONAL COMPLEXITY

The above algorithm requires two calls to $f$.

\section{General monotoniC SITUATION (WITH UNKNOWN DIRECTIONS OF MONOTONICITY): DESCRIPTION}

In more complex situations, when the function $f\left(x_{1}, \ldots, x_{n}\right)$ is not an explicit expression but rather a complex algorithm, we may still know that the dependence is monotonic, but we do not know a priori with respect to which variables the dependence is increasing and with respect to which the dependence is decreasing.

\section{GENERAL MONOTONIC SITUATION: ANALYSIS}

In this case, for each $i$, we can find the corresponding direction of monotonicity $\varepsilon_{i}$ by changing the value of the $i$ th input and tracing how this change will affect the resulting value $f$ :

$$
\varepsilon_{i}=\operatorname{sign}\left(f\left(\widetilde{x}_{1}, \ldots, \widetilde{x}_{i-1}, \widetilde{x}_{i}+h_{i}, \widetilde{x}_{i+1}, \ldots, \widetilde{x}_{n}\right)-\widetilde{y}\right)
$$

for some small value $h_{i}>0$; see, e.g., [6], [12], [13] and references therein. Thus, we arrive at the following algorithm. 
XVII. GENER AL MONOTONIC SITUATION: ALGORITHM

- First, we compute $\widetilde{y}=f\left(\widetilde{x}_{1}, \ldots, \widetilde{x}_{n}\right)$.

- Second, for all $i$ from 1 to $n$, we compute $f\left(\widetilde{x}_{1}, \ldots, \widetilde{x}_{i-1}, \widetilde{x}_{i}+h_{i}, \widetilde{x}_{i+1}, \ldots, \widetilde{x}_{n}\right)$ for some small value $h_{i}>0$ and then find

$$
\varepsilon_{i}=\operatorname{sign}\left(f\left(\widetilde{x}_{1}, \ldots, \widetilde{x}_{i-1}, \widetilde{x}_{i}+h_{i}, \widetilde{x}_{i+1}, \ldots, \widetilde{x}_{n}\right)-\widetilde{y}\right) .
$$

- Finally, we compute the range $[\underline{y}, \bar{y}]$ by using the formulas

$$
\begin{aligned}
& \underline{y}=f\left(\widetilde{x}_{1}-\varepsilon_{1} \cdot \Delta_{1}, \ldots, \widetilde{x}_{n}-\varepsilon_{n} \cdot \Delta_{n}\right) \\
& \bar{y}=f\left(\widetilde{x}_{1}+\varepsilon_{1} \cdot \Delta_{1}, \ldots, \widetilde{x}_{n}+\varepsilon_{n} \cdot \Delta_{n}\right) .
\end{aligned}
$$

\section{GENERAL MONOTONIC SITUATION: COMPUTATIONAL COMPLEXITY}

In this case, in addition to the original call to $f$ for computing $\widetilde{y}=f\left(\widetilde{x}_{1}, \ldots, \widetilde{x}_{n}\right)$, we need $n$ calls to find $n$ signs $\varepsilon_{i}$, and then 2 more calls to compute $\underline{y}$ and $\bar{y}$. Overall, we thus need $n+3$ calls to $f$.

XIX. Situation When the algorithm $f\left(x_{1}, \ldots, x_{n}\right)$ IS A SOLUTION TO A SYSTEM OF EQUATIONS: DESCRIPTION

We have mentioned that in many cases, the complex algorithm $f\left(x_{1}, \ldots, x_{n}\right)$ comes from the need to solve a difficultto-solve non-linear system of equations. In such situations, the desired quantity $y$ is one of several unknowns $y_{1}=y, y_{2}$, $\ldots, y_{m}$ which are related to the easier-to-estimate values by a system of non-linear equations

$$
\begin{gathered}
F_{1}\left(x_{1}, \ldots, x_{n}, y_{1}, \ldots, y_{m}\right)=0 \\
\ldots \\
F_{m}\left(x_{1}, \ldots, x_{n}, y_{1}, \ldots, y_{m}\right)=0 .
\end{gathered}
$$

Often, the functions $F_{1}, \ldots, F_{m}$ are reasonably easy to compute, it is the solution which requires a large amount of time.

We start with the values $\widetilde{y}_{1}, \ldots, \widetilde{y}_{m}$ which correspond to the (approximate) estimated values $\widetilde{x}_{1}, \ldots, \widetilde{x}_{n}$ of the inputs. For these values, the following system of equations holds:

$$
\begin{gathered}
F_{1}\left(\widetilde{x}_{1}, \ldots, \widetilde{x}_{n}, \widetilde{y}_{1}, \ldots, \widetilde{y}_{m}\right)=0 \\
\ldots \\
F_{m}\left(\widetilde{x}_{1}, \ldots, \widetilde{x}_{n}, \widetilde{y}_{1}, \ldots, \widetilde{y}_{m}\right)=0 .
\end{gathered}
$$

We want to know how the uncertainty $\Delta x_{i}$ in the inputs affects the uncertainty $\Delta y_{j}$ in the outputs, i.e., how the inputs $\Delta x_{i}$ affect the values $\Delta y_{j}$ for which

$$
\begin{gathered}
F_{1}\left(\widetilde{x}_{1}-\Delta x_{1}, \ldots, \widetilde{x}_{n}-\Delta x_{n}, \widetilde{y}_{1}-\Delta y_{1}, \ldots, \widetilde{y}_{m}-\Delta y_{m}\right)=0 ; \\
\ldots \\
F_{m}\left(\widetilde{x}_{1}-\Delta x_{1}, \ldots, \widetilde{x}_{n}-\Delta x_{n}, \widetilde{y}_{1}-\Delta y_{1}, \ldots, \widetilde{y}_{m}-\Delta y_{m}\right)=0 .
\end{gathered}
$$

In this case, e.g., the above linearization algorithm means that we need to call $f n+1$ times means that we need to solve difficult-to-solve non-linear system of equations $n+3$ times. A natural question is: can we estimate the range $[\underline{y}, \bar{y}]$ faster, without actually having to solve that many systems of non-linear equations?

In this paper, we will show that such a speed-up is indeed possible. Preliminary speed-up results were described in [12], [13].

\section{SYSTEMS OF EQUATIONS, LINEARIZATION CASE: ANALYSIS}

First, we will consider the case when the functions $F_{k}$ can be safely linearized, i.e., when the terms quadratic in $\Delta x_{i}$ and in $\Delta y_{j}$ can be safely ignored. In this case, the above equations lead to

$$
\sum_{i=1}^{n} X_{k i} \cdot \Delta x_{i}+\sum_{j=1}^{m} Y_{k j} \cdot \Delta y_{j}=0
$$

where we denoted

$$
X_{k i} \stackrel{\text { def }}{=} \frac{\partial F_{k}}{\partial x_{i}}, \quad Y_{k j} \stackrel{\text { def }}{=} \frac{\partial F_{k}}{\partial y_{j}} .
$$

Since the functions $F_{k}$ are easy-to-compute, the computation of these partial derivatives does not require the timeconsuming step of actually solving the non-linear system of equations. The above equations can be described in a matrix form: $X \Delta x=-Y \Delta y$. Thus, we can find the vector $\Delta y$ as $\Delta y=-Y^{-1} X \Delta x$, i.e., as $\Delta y=-M \Delta x$, where the matrix $M$ with elements $m_{j i}$ has the form $M=Y^{-1} X$.

In this case,

$$
\Delta y_{j}=\sum_{i=1}^{n} m_{j i} \cdot \Delta x_{i} .
$$

In particular, for the desired quantity $y=y_{1}$, we get

$$
\Delta y_{1}=\sum_{i=1}^{n} m_{1 i} \cdot \Delta x_{i},
$$

and thus, the interval of possible values of $\Delta y$ is $[-\Delta, \Delta]$, where

$$
\Delta=\sum_{i=1}^{n}\left|m_{1 i}\right| \cdot \Delta_{i}
$$

Thus, we arrive at the following algorithm.

\section{SySTEMS OF EQUATIONS, LINEARIZATION CASE: ALGORITHM}

- First, we use the estimates $\widetilde{x}_{1}, \ldots, \widetilde{x}_{n}$ to solve the original system of non-linear equations

$$
\begin{gathered}
F_{1}\left(\widetilde{x}_{1}, \ldots, \widetilde{x}_{n}, \widetilde{y}_{1}, \ldots, \widetilde{y}_{m}\right)=0 \\
\ldots \\
F_{m}\left(\widetilde{x}_{1}, \ldots, \widetilde{x}_{n}, \widetilde{y}_{1}, \ldots, \widetilde{y}_{m}\right)=0
\end{gathered}
$$

and find the values $\widetilde{y}_{1}=\widetilde{y}, \widetilde{y}_{2} \ldots, \widetilde{y}_{m}$.

- Then, we compute the derivative matrices with elements

$$
X_{k i} \stackrel{\text { def }}{=} \frac{\partial F_{k}}{\partial x_{i}}, \quad Y_{k j} \stackrel{\text { def }}{=} \frac{\partial F_{k}}{\partial y_{j}},
$$

by either analytically differentiating or by using numerical differentiation. 
- Third, we compute the matrix $M=Y^{-1} X$ with elements $m_{j i}$, the bound

$$
\Delta=\sum_{i=1}^{n}\left|m_{1 i}\right| \cdot \Delta_{i},
$$

and the desired range $[\widetilde{y}-\Delta, \widetilde{y}+\Delta]$.

\section{SYSTEMS OF EQUATIONS, LINEARIZATION CASE: COMPUTATIONAL COMPLEXITY}

According to the above algorithm, we can compute the desired bound $\Delta$ by solving the original system of non-linear equations only once: to find the original values $\widetilde{y}_{j}$.

Comment. This is clearly much faster than solving this system $n+1$ times. There is a computational advantage of using this technique even if the original system of equations is linear: in this case, instead of solving the system of equations $n+1$ times, we compute the inverse matrix once. It is known that asymptotically, the computation time needed to invert a matrix $A$ is the same as the time needed to solve a general linear equation $A x=b$ (see, e.g., [1]), so this idea indeed leads to a drastic decrease in computation time.

\section{SYSTEM OF EQUATIONS, MONOTONIC CASE: ANALYSIS}

If we know that the dependence of $y$ on each $x_{i}$ is monotonic, but we do not know the direction of monotonicity, then we can find this direction by computing the sign $\varepsilon_{i}$ of the partial derivative

$$
m_{1 i}=\frac{\partial y_{1}}{\partial x_{i}}:
$$

$\varepsilon_{i}=\operatorname{sign}\left(m_{1 i}\right)$. Similarly to the linearized case, we can conclude that the matrix $M$ with elements $m_{k i}=\frac{\partial y_{k}}{\partial x_{i}}$ can be computed as $M=Y^{-1} X$. Computing the partial derivatives $X$ and $Y$ does not require solving any system of non-linear equations. Thus, we arrive at the following algorithm.

\section{SYSTEM OF EQUATIONS, MONOTONIC CASE: ALGORITHM}

- First, we use the estimates $\widetilde{x}_{1}, \ldots, \widetilde{x}_{n}$ to solve the original system of non-linear equations

$$
\begin{gathered}
F_{1}\left(\widetilde{x}_{1}, \ldots, \widetilde{x}_{n}, \widetilde{y}_{1}, \ldots, \widetilde{y}_{m}\right)=0 ; \\
\ldots \\
F_{m}\left(\widetilde{x}_{1}, \ldots, \widetilde{x}_{n}, \widetilde{y}_{1}, \ldots, \widetilde{y}_{m}\right)=0 ;
\end{gathered}
$$

and find the values $\widetilde{y}_{1}=\widetilde{y}, \widetilde{y}_{2} \ldots, \widetilde{y}_{m}$.

- Then, we compute the derivative matrices with elements

$$
X_{k i} \stackrel{\text { def }}{=} \frac{\partial F_{k}}{\partial x_{i}}, \quad Y_{k j} \stackrel{\text { def }}{=} \frac{\partial F_{k}}{\partial y_{j}},
$$

by either analytically differentiating or by using numerical differentiation.

- Third, we compute the matrix $M=Y^{-1} X$ with elements $m_{j i}$, and the signs $\varepsilon_{i}=\operatorname{sign}\left(m_{1 i}\right)$.
- To find $y$, we solve the system

$$
\begin{gathered}
F_{1}\left(\widetilde{x}_{1}-\varepsilon_{1} \cdot \Delta_{1}, \ldots, \widetilde{x}_{n}-\varepsilon_{n} \cdot \Delta_{n}, y_{1}, \ldots, y_{m}\right)=0 ; \\
\ldots \\
F_{m}\left(\widetilde{x}_{1}-\varepsilon_{1} \cdot \Delta_{1}, \ldots, \widetilde{x}_{n}-\varepsilon_{n} \cdot \Delta_{n}, y_{1}, \ldots, y_{m}\right)=0 ;
\end{gathered}
$$

and take $\underline{y}=y_{1}$.

- To find $\bar{y}$, we solve the system

$$
\begin{gathered}
F_{1}\left(\widetilde{x}_{1}+\varepsilon_{1} \cdot \Delta_{1}, \ldots, \widetilde{x}_{n}+\varepsilon_{n} \cdot \Delta_{n}, y_{1}, \ldots, y_{m}\right)=0 ; \\
\ldots \\
F_{m}\left(\widetilde{x}_{1}+\varepsilon_{1} \cdot \Delta_{1}, \ldots, \widetilde{x}_{n}+\varepsilon_{n} \cdot \Delta_{n}, y_{1}, \ldots, y_{m}\right)=0 ;
\end{gathered}
$$

and take $\bar{y}=y_{1}$. The desired range is now $[\underline{y}, \bar{y}]$.

\section{SySTEM OF EQUATIONS, MONOTONIC CASE: COMPUTATIONAL COMPLEXITY}

Thus, in the monotonic case, we can find the range of $y$ by solving only 3 systems:

- first, we solve the system corresponding to the original estimates $\widetilde{x}_{i}$ and to find the original values $\widetilde{y}_{j}$; after this, we compute $X, Y, M$, and $\varepsilon_{i}$;

- second, we solve the system corresponding to the values $x_{i}=\widetilde{x}_{i}+\varepsilon_{i} \cdot \Delta_{i}$ and thus, compute $\bar{y}$ as the corresponding $y_{1}$;

- finally, we solve the system corresponding to the values $x_{i}=\widetilde{x}_{i}-\varepsilon_{i} \cdot \Delta_{i}$ and thus, compute $\underline{y}$ as the corresponding $y_{1}$.

Solving 3 systems takes longer than solving a single system (as in the linearized case), but it is much faster than solving $n+3$ systems according to the general monotonicity case.

\section{CASE OF UNKNOWN FUNCTIONAL VARIABLES}

In some practical situations, instead of the values $x_{1}, \ldots, x_{n}$ known with interval uncertainty, we have a function $x(t)$ known with interval uncertainty, i.e., we know the approximate function $\widetilde{x}(t)$ and we know the bounds $\Delta(t)$ on the approximation error. In other words, we know that for each $t$, the (unknown) actual value $x(t)$ belongs to the interval $[\widetilde{x}(t)-\Delta(t), \widetilde{x}(t)+\Delta(t)]$.

The desired value $y$ is related to the function $y$ by a known functional dependence $y=F(x)$ : e.g., we may have

$$
y=y_{0}+\int a(t) \cdot x(t) d t
$$

or

$$
y=y_{0}+\int a(t) \cdot x(t) d t+\int a(t, s) \cdot x(t) \cdot x(s) d t d s .
$$

In general, we have $\widetilde{y}=F(\widetilde{x})$ and $y=F(x)=F(\widetilde{x}-\Delta x)$, where $\Delta x(t) \stackrel{\text { def }}{=} \widetilde{x}(t)-x(t)$.

When the approximation errors $\Delta x(t)$ are small, we can expand the dependence $y=F(\widetilde{x}-\Delta x)$ or $\Delta x$ in Taylor series and ignore quadratic and higher order terms in this dependence. In this case, we conclude that

$$
\Delta y=\int \frac{\delta y}{\delta x(t)} \cdot \Delta x(t) d t
$$


where $\frac{\delta y}{\delta x(t)}$ is a functional derivative. For example,

- For a linear functional $y=y_{0}+\int a(t) \cdot(\widetilde{x}(t)-\Delta x(t)) d t$, we have $\frac{\delta y}{\delta x(t)}=a(t)$.

- For a quadratic functional

$$
\begin{gathered}
y=y_{0}+\int a(t) \cdot(\widetilde{x}(t)-\Delta x(t)) d t+ \\
\int a(t, s) \cdot(\widetilde{x}(t)-\Delta x(t)) \cdot(\widetilde{x}(s)-\Delta x(s)) d t d s= \\
\left(y_{0}+\int a(t) \cdot \widetilde{x}(t) d t+\int a(t, s) \cdot \widetilde{x}(t) \cdot \widetilde{x}(s) d t d s\right)- \\
\left.\int a(t) \cdot \Delta x(t)\right) d t-2 \int a(t, s) \cdot \widetilde{x}(s) \cdot \Delta x(t) d t d s, \\
\text { we have } \frac{\delta y}{\delta x(t)}=a(t)+2 \int a(t, s) \cdot \widetilde{x}(s) d s .
\end{gathered}
$$

In this case, the largest possible value $\Delta$ of $\Delta t$ is attained when

$$
\begin{aligned}
& \text { - } \Delta x(t)=\Delta(t) \text { for all } t \text { with } \frac{\delta y}{\delta x(t)} \geq 0 \text {, and } \\
& \text { - } \Delta x(t)=-\Delta(t) \text { for all } t \text { with } \frac{\delta y}{\delta x(t)}<0 .
\end{aligned}
$$

In other words, the range of possible values of $y$ is

$$
[\widetilde{y}-\Delta, \widetilde{y}+\Delta], \text { where } \Delta=\int\left|\frac{\delta y}{\delta x(t)}\right| \cdot \Delta(t) d t .
$$

\section{CASE OF UNCERTAIN BOUNDARY}

In some practical problems, e.g., in many problems related to structural integrity, the desired quantity $y$ is related to the solution $u$ of a linear partial differential equation $L u=f$ with a known right-hand side $f$ and boundary conditions such as $u_{\mid \partial \Omega}=0$.

In numerical mathematics, it is usually assumed that we know the domain $\Omega$ and its boundary $\partial \Omega$. However, in practice, we often only know the boundary $\partial \Omega$ with uncertainty. How does this uncertainty affect the solution $u$ to the corresponding partial differential equation?

In this paper, we will consider this effect only for the linearized case, when the uncertainty in $\partial \Omega$ is small, and we can safely ignore terms which are quadratic and of higher order in terms of this uncertainty.

Our main idea is to transform the boundary condition corresponding into the actual (unknown) domain $\Omega$ to the boundary condition corresponding to the nominal (approximate) domain $\widetilde{\Omega}$. Let $\widetilde{u}$ be the solution corresponding to $\Omega$. We are interested in the difference $\Delta u=\widetilde{u}-u$. For each point $y \in \partial \widetilde{\Omega}$, let $\rho(y)$ denote the distance from this point to the nearest point on the boundary $\partial \Omega$. In these terms, the closeness between the sets means, e.g., that we have an upper bound $\Delta_{\Omega}(y)$ on this distance.

Let us describe this closest point. Let $\varepsilon(y)=1$ if the nearest point is outside $\widetilde{\Omega}$ and $\varepsilon(y)=-1$ if the nearest point is inside $\widetilde{\Omega}$. Let $\vec{n}$ be a unit vector orthogonal to $\partial \widetilde{\Omega}$ and directed outside $\widetilde{\Omega}$. Then, in the linear approximation, the closest point is equal to $y+\varepsilon(y) \cdot \rho(y) \cdot \vec{n}$, and the value of $u$ at this closest point is equal to $u(y+\varepsilon(y) \cdot \rho(y) \cdot \vec{n})=u(y)+\varepsilon(y) \cdot \rho(y) \cdot \frac{d u}{d n}$. By definition, $u(y)=\widetilde{u}(y)+\Delta u(y)$. For $y \in \delta \widetilde{\Omega}$, we have $\widetilde{u}(y)=$ 0 , and in the term proportional to $\frac{d u}{d n}$, the part proportional to $\Delta u$ can be ignored. As a result, we get the boundary condition $\Delta u_{\mid \delta \widetilde{\Omega}}=g$, where $g(y) \stackrel{\text { def }}{=} \varepsilon(y) \cdot \rho(y) \cdot \frac{d \widetilde{u}}{d n}(y)$.

Since $L$ is a linear operator, from $L u=f$ and $L \widetilde{u}=f$, we conclude that $L \Delta u=0$. In general, the solution to a linear problem $L v=0, v_{\mid \delta \widetilde{\Omega}}=g$ is linear in $g$, i.e., $v(x)=$ $\int a(x, y) \cdot g(y) d y$ for some function $a(x, y)$. Substituting the above expression for $g(y)$ into this formula, and taking into account that $\rho(y) \leq \Delta_{\Omega}(y)$, we conclude that for every $x$, we have

$$
|\Delta u(x)| \leq \Delta_{u}(x) \stackrel{\text { def }}{=} \int|a(x, y)| \cdot \Delta_{\Omega}(y) \cdot\left|\frac{d \widetilde{u}}{d n}(y)\right| d y .
$$

Comments. If we have uncertainty both in $f$ and in $\Omega$, then we can simply add the resulting bounds on $\Delta u(x)$.

A similar idea can be also applied to the case of nonlinear equations $L$, the only difference is that we will need to linearize the operator $L$.

\section{ACKNOWLEDGMENTS}

The authors are thankful to the anonymous referees for valuable discussions.

\section{REFERENCES}

[1] Th. H. Cormen, C. E. Leiserson, R. L. Rivest, and C. Stein, Introduction to Algorithms, MIT Press, Cambridge, MA, 2001.

[2] D. Dubois and H. Prade, Operations on fuzzy numbers, International Journal of Systems Science, 1978, Vol. 9, pp. 613-626.

[3] Interval computations website http://www.cs.utep.edu/interval-comp

[4] L. Jaulin, M. Kieffer, O. Didrit, and E. Walter, Applied Interval Analysis, with Examples in Parameter and State Estimation, Robust Control and Robotics, Springer-Verlag, London, 2001.

[5] G. Klir and B. Yuan, Fuzzy sets and fuzzy logic, Prentice Hall, New Jersey, 1995.

[6] V. Kreinovich, J. Beck, C. Ferregut, A. Sanchez, G. R. Keller, M. Averill, and S. A. Starks, "Monte-Carlo-type techniques for processing interval uncertainty, and their potential engineering applications", Reliable Computing, 2007, Vol. 13, No. 1, pp. 25-69.

[7] V. Kreinovich and S. Ferson, "A New Cauchy-Based Black-Box Technique for Uncertainty in Risk Analysis", Reliability Engineering and Systems Safety, 2004, Vol. 85, No. 1-3, pp. 267-279.

[8] V. Kreinovich, A. Lakeyev, J. Rohn, and P. Kahl, Computational complexity and feasibility of data processing and interval computations, Kluwer, Dordrecht, 1998.

[9] R. E. Moore and W. Lodwick, Interval Analysis and Fuzzy Set Theory, Fuzzy Sets and Systems, 2003, Vol. 135, No. 1, pp. 5-9.

[10] H. T. Nguyen and V. Kreinovich, Nested Intervals and Sets: Concepts, Relations to Fuzzy Sets, and Applications, In: R. B. Kearfott and V. Kreinovich, eds., Applications of Interval Computations, Kluwer, Dordrecht, 1996, pp. 245-290.

[11] H. T. Nguyen and E. A. Walker, A First Course in Fuzzy Logic, CRC Press, Boca Raton, Florida, 2006.

[12] A. Pownuk, "General Interval FEM Program Based on Sensitivity Analysis", Proceedings of the NSF workshop on Reliable Engineering Computing, Savannah, Georgia, February 20-22, 2008.

[13] M. V. Rama Rao, A. Pownuk, and I. Skalna, "Stress Analysis of a Singly Reinforced Concrete Beam with Uncertain Structural Parameters", Proceedings of the NSF workshop on Reliable Engineering Computing, Savannah, Georgia, February 20-22, 2008.

[14] S. Rabinovich, Measurement Errors and Uncertainties: Theory and Practice, American Institute of Physics, New York, NY, 2005. 